\title{
METODOLOGÍA DEL DERECHO COMPARADO \\ EN DERECHOS HUMANOS
}

\section{METHODOLOGY OF COMPARATIVE LAW ON HUMAN RIGHTS}

\author{
José Caramelo Gomes \\ Maria Manuela Magalhães Silva \\ Universidad Portucalense
}

\begin{abstract}
Resumen: Este artículo presenta los métodos de Derecho Comparado más comunes en la actualidad y su aplicación al dominio de los Derechos Humanos.

Palabras-clave: Derecho Comparado, Método Científico, Metodología Conceptual, Metodología de Caso, Metodología funcional, Tertium comparationis, Derechos Humanos.
\end{abstract}

\begin{abstract}
:
This article presents and discusses the use of Comparative Law Methods in the field of Human Rights.

Keywords: Comparative Law, Scientific method, Conceptual method, Case method, Functional method, Tertium comparationis, Human rights.
\end{abstract}

\section{Introducción y objetivos del Derecho Comparado moderno}

Tenemos una variedad de distintos propósitos para la disciplina de Derecho Comparado que, como dice Sacco ${ }^{1}$, parte del presupuesto que hay una multiplicidad de soluciones normativas para una misma función, problema, concepto o situación: "Comparative law presupposes the existence of a plurality of legal rules and institutions. It studies them in order to establish to what extent they are identical or different".

Es tradición, en Iberia, que los estudios e investigaciones en ciencia jurídica tengan una sección dedicada al Derecho Comparado. Los investigadores al realizar el estado del arte sobre este tema, en general, seleccionan una bibliografía sobre el ordenamiento en análisis, hacen una revisión de literatura e realizan las conclusiones en esa sección. La elección de los ordenamientos se basa, normalmente, en un criterio pragmático: si el investigador comprende el idioma en el que la fuente está escrita. Así, es habitual la "comparación" entre el derecho nacional de países en que el idioma es el castellano o el portugués, y pocas veces se realiza la "comparación" con el

\footnotetext{
${ }^{1}$ Sacco, Rodolfo. "Legal Formants, A Dynamic Approach to Comparative Law". American Journal of Comparative Law, 1991.
} 
derecho escrito en francés o inglés, o incluso, en alemán (en este caso frecuentemente utilizando traducciones o fuentes secundarias). En realidad, este sistema o metodología está muy distante de la moderna metodología del Derecho Comparado y puede ser la razón por la cual la academia jurídica ibérica se queda, muchas veces, fuera de los grandes proyectos de investigación en ciencia jurídica que son financiados por la Unión Europea.

Esta comunicación tiene el objetivo de iniciar una discusión, en Iberia, sobre una mejor utilización de las técnicas modernas del Derecho Comparado en la academia jurídica latino-ibero-americana, en particular en lo que respecta a la investigación en Derechos Humanos.

El Derecho comparado es una Ciencia Jurídica que, como ciencia, tiene un método científico, o sea, un protocolo que comanda el procedimiento de investigación y que permite, en futuro, a través de la repetición (repetitividad), y la validación de las conclusiones obtenidas, garantizando así su naturaleza científica.

Cualquier ciencia tiene un objeto. El del Derecho Comparado es el Derecho, como fenómeno social. Pero eso no es suficiente, ya que eso es el objeto de toda a Ciencia Jurídica. Tiene que haber algo más; y ese algo más es la comparación, o método comparativo, que puede ser aplicado en distintas cuestiones, hechos, conceptos, conflictos y funciones.

La aplicación que se hará del método comparativo es determinante para el método de recoger la información necesaria, y eso es lo que diferencia los distintos métodos del Derecho Comparado que más adelante se incluyen. Por ahora, vamos a clarificar un poco lo que es y cómo se desarrolló el Derecho Comparado actual.

Durante los siglos XIX y XX era común la idea de que el Derecho Comparado debería tener objetivos específicos. El punto de partida de esta idea es que el derecho comparado es diferente del Derecho, del cual sería meramente subsidiario. Este punto de vista ha cambiado y ahora el Derecho Comparado se integra cada vez más en el estudio del derecho, como un apoyo técnico o una metodología de investigación avanzada. Estos dos enfoques han estado históricamente presentes a lo largo de los siglos, desde los estudios jurídicos de la antigua Grecia hasta la actualidad. El uso del derecho comparado como un instrumento de reforma legislativa, es evidente en la comparación de las constituciones hechas por Aristóteles, 
al igual que la influencia de la "visita de estudio" romana en Grecia, en el proceso elaboración de la Ley de las Doce Tablas ${ }^{2}$.

Durante el siglo XIX y en parte durante el siglo XX, un período durante el cual se desarrolla el derecho comparado contemporáneo, el darwinismo social ha condicionado su creación y evolución como una disciplina evolucionista. Sin embargo, otro de los objetivos era establecer una taxonomía de las leyes del mundo según diversas nociones de familias jurídicas o tipos ideales de leyes, bajo la perspectiva de la construcción occidental, en la senda de Max Weber. Esta idea de la clasificación del Derecho del mundo, así como la de la evolución jurídica, hoy ha perdido gran parte de su interés y asistimos más recientemente a un regreso de la concepción utilitaria y constructivista ya presente en la utilización dada por Aristóteles ${ }^{3}$.

La concepción constructivista lleva el Derecho Comparado a ser un instrumento para la mejoría del Derecho, por la comparación con otras soluciones de distintas órdenes jurídicas o de distintas épocas. Así, es posible encontrar ejemplos de utilización en los sistemas de derecho continental (originando algunas veces, el trasplante jurídico, con todos los problemas del "rechazo genético"), o en los sistemas de "common law" como un instrumento de mejoría de la jurisprudencia ${ }^{4}$.

Al mismo tiempo, el Derecho Comparado empezó a cumplir un objetivo mucho más importante, la armonización de Derechos. Esa es, en realidad, la función más importante de la disciplina en la actualidad ${ }^{5} \mathrm{y}$, como escribió Glenn, una forma de adjuntar lo que es diferente manteniendo las diferencias.

\section{Metodología del Derecho Comparado}

El método científico aplicado al Derecho Comparado contiene varias fases: la delimitación del fenómeno jurídico que se va a estudiar, los ordenamientos en los que se va hacer y porqué esos y no otros (y aquí hay que considerar que se pueden estudiar distintos países, distintos ordenamientos, o un único ordenamiento jurídico en distintas épocas, por ejemplo), y para que servirá el estudio - para conocer y

\footnotetext{
${ }^{2}$ Glenn, H. Patrick. "Aims of comparative law". In: Smits, Jan M., (ed.). Elgar Encyclopedia of Comparative Law, Cheltenham: Elgar Publishing; 2014.

${ }^{3}$ Ibidem.

${ }^{4}$ Ibidem

${ }^{5}$ Van der Mensbrugghe, François. L'utilisation de la méthode comparative en droit européen. Namur: Presses universitaires de Namur, 2003.
} 
comparar conceptos jurídicos, para conocer soluciones para un litigio, para conocer cómo se cumple una función o un objetivo jurídico). La fase siguiente será la elección de la metodología adecuada para la recogida de la información en el formato necesario para la comparación final, la definición de redundancias y metodologías redundantes, para la validación, y, finalmente, la definición de los criterios de evaluación de las recomendaciones extraidas de las conclusiones, cuando aplicable. O, en síntesis, como señala Pieters ${ }^{6}$ refiriendo a Constantinesco, "hay tres fases de la comparación de derecho: Conocer, comprender y comparar. De alguna una forma estas tres fases deberán de hecho estar presente en cualquier comparación. Se tiene que reunir información sobre el objeto de investigación comparativa, se tiene que reflejar lo que se encuentra en los diversos órdenes legales dentro de su contexto nacional, y se tiene que realizar la comparación de los mismos, es decir, establecer la similitud y diferencias entre lo que se ha identificado en los diversos órdenes de comparación."

\subsection{La metodología conceptual}

El enfoque tradicional de la metodología comparativa son las reglas formales, comparadas con independencia de la cultura y otros formantes que las rodean ${ }^{7}$. Para ello, el comparativista define el tertium comparationis, es decir, con qué comparar, definir las órdenes legales involucradas, donde comparar, buscar las normas legales pertinentes, interpretado de acuerdo a las reglas de interpretación de la ley y comparar los resultados de ambos elementos de regla: la descripción de los hechos y el comando. Esta metodología presenta varios problemas y limitaciones, siendo el primero el obstáculo del idioma. Cómo David señala ${ }^{8}$, traducir los términos legales no es en absoluto necesario, ya que la ley se compone esencialmente de conceptos abstractos: es fácil encontrar la palabra equivalente para silla en todos los idiomas; señalas una silla y los nativos dan rápidamente la respuesta. Cuando se habla de la ley las cosas son muy diferentes. No hay manera para que apunte al derecho de

\footnotetext{
${ }^{6}$ Pieters, D. "Functions of comparative law and practical methodology of comparing". Syllabus Research Master in Law. 2009.

${ }^{7}$ Legrand, Pierre. Sens et non-sens d'un Code civil européen. RIDC. 1996, pp.779-812.

${ }^{8}$ David, René. Brierley, John, E. Major legal systems in the world today: an introduction to the comparative study of law: Simon and Schuster. s.d. 1978.
} 
propiedad y obtener la palabra equivalente para ello: el contenido del concepto puede variar significativamente.

Por otra parte, las abordajes posmodernistas a la metodología de derecho comparado, tales como los de Legran ${ }^{9}$ y Jaime ${ }^{10}$, evidencia la necesidad de replantear mediante la inclusión del entorno cultural, social y económico de la ley y del investigador con el fin de obtener conclusiones válidas a partir de la comparación. El problema es que Jayme no presenta una solución para hacerlo y Legran ${ }^{11}$ simplemente no lo considera posible ${ }^{12}$.

La crítica de Legrand y Jayme a la metodología comparativa se puede dividir en dos aspectos diferentes: el contexto del investigador y el contexto de la ley, considerando contexto como la totalidad de los aspectos culturales, sociales, económicos y ambiente jurídico. En última instancia, estos requisitos implican que el comparatista debe i) estar al mismo nivel de competencia de lengua materna como en el idioma de las diversas leyes bajo comparación que se escriben; ii) tener una formación jurídica completa e inmersiva en la ley en todos los órdenes legales que se comparan. Esta idea de la necesidad de una metodología comparativa está presente incluso en alguna jurisprudencia del Tribunal Europeo de Justicia de la Comunidad (caso Cilfit, la doctrina de acto claro). El caso Cilfit (Asunto 283/81, CILFIT y otros [1982] ECR, disponible en http://curia.eu.int) consistio en una referencia para el TJCE en el antiguo artículo 177 del Tratado CE, por la primera sala civil la Corte suprema di cassazione (Tribunal Supremo de Casación) de decisión prejudicial, sobre la interpretación del tercer párrafo del mismo artículo, i. e., la obligación de los tribunales nacionales para abordar cuestiones prejudiciales en la interpretación del derecho comunitario al TJCE.

La cuestión de la Corte Suprema di Cassazione era «El párrafo tercero del artículo 177 del Tratado, que dispone que, cuando se plantee una cuestión de las que enumera el párrafo primero del mismo artículo, en un litigio pendiente ante un órgano jurisdiccional nacional, cuyas decisiones no son susceptibles de un ulterior recurso judicial de Derecho interno, dicho órgano estará obligado a someter la cuestión al

\footnotetext{
${ }^{9}$ Legrand, Pierre. Sens et non-sens..., pp.779-812.

${ }^{10}$ Jayme, Erik. "Identité culturelle et intégration: Le Droit international privé postmoderne". Recueil des Cours, Hague Academy of International Law, 1995.

${ }_{11}^{11}$ Legrand, Pierre. Sens et non-sens..., pp. 779-812.

${ }^{12}$ ERP, J. European Private Law Postmodern Dilemmas and Choices, towards a Method of Adequate Comparative Legal Analysis. EJCL, 1999.
} 
Tribunal de Justicia, ¿establece una obligación de plantear una cuestión prejudicial que no permite al Juez nacional llevar a cabo apreciación alguna acerca del fundamento de la cuestión suscitada, o bien condiciona dicha obligación y, en su caso, dentro de qué límites, a la existencia previa de una duda de interpretación razonable?».

El vínculo entre esta cuestión y Derecho comparado es que la respuesta del Tribunal de Justicia sigue la crítica posmodernista con el método comparativo y lo hace de circunstancias especialmente favorables: la norma bajo análisis se incluyó en el Reglamento CE, así, con contenidos presumiblemente similares a través de la CE. Era una cuestión de comparar la misma ley en diferentes versiones lingüísticas.

En referencia al proceso de determinación de la correcta interpretación del reglamento de la CE, el Tribunal de Justicia señaló que implica el análisis de las diferentes versiones del lingüista de la misma norma teniendo en cuenta que cada una de estas versiones se han de interpretar por los juristas con la educación legal en el contexto social, cultural y económico específica:

16. Finalmente, la correcta aplicación del Derecho comunitario puede imponerse con tal evidencia que no deje lugar a duda razonable alguna sobre la solución de la cuestión suscitada. Antes de concluir que se da tal situación, el órgano jurisdiccional nacional debe llegar a la convicción de que la misma evidencia se impondría igualmente a los órganos jurisdiccionales nacionales de los otros Estados miembros, así como al Tribunal de Justicia. Tan solo si estas condiciones se reúnen puede abstenerse el órgano jurisdiccional nacional de someter la cuestión al Tribunal de Justicia y resolver bajo su propia responsabilidad.

17. No obstante, la existencia de tal posibilidad debe ser apreciada en función de las características del Derecho comunitario y de las dificultades particulares que presenta su interpretación.

18. Debe, en primer lugar, tenerse en cuenta que los textos de Derecho comunitario están redactados en varias lenguas y que las diversas versiones lingüísticas son auténticas por igual; por tanto, la interpretación de una disposición de Derecho comunitario supone una comparación de las versiones lingüísticas.

19. Debe observarse a continuación que, incluso en caso de exacta concordancia de las versiones lingüísticas, el Derecho comunitario utiliza una terminología propia. Por lo demás, ha de subrayarse que los conceptos jurídicos no tienen necesariamente el mismo contenido en el Derecho comunitario y en los diferentes Derechos nacionales. 
20. Finalmente, cada disposición de Derecho comunitario debe ser situada en su contexto e interpretada a la luz del conjunto de las disposiciones de ese Derecho, de sus finalidades y de su grado de evolución en la fecha en la que debe hacerse aplicación de la disposición de que se trata.

\subsection{La metodología factual o de caso}

Esta crítica es de alguna manera superada por el proyecto de Cornell, dirigido por R. Schlesinger en la década de 1960. El problema inicial que Schlesinger tuvo que resolver era cómo obtener información comparables a las preguntas sobre los diferentes sistemas jurídicos. Las respuestas tenían que referirse a las preguntas idénticas interpretadas de forma idéntica por todos los que respondieron. Schlesinger ${ }^{13}$ ha formulado cada pregunta para tener en cuenta cualquier circunstancia pertinente en cualquiera de los sistemas analizados, así permitiendo la comparabilidad. Por lo tanto, la característica especial de los trabajos realizados en Cornell es que cada pregunta se formuló mediante la presentación de un caso práctico.

Hay dos problemas principales con la metodología de Schlesinger. El primero es el que Sacco ${ }^{14}$ señala, que la lista, incluso exhaustiva, de todas las razones dadas por las decisiones tomadas por los tribunales no es toda la ley, los estatutos no son toda la ley, ni tampoco las definiciones de las doctrinas de derecho por los estudiosos lo son. Con el fin de saber cuál es la ley, es necesario analizar toda la compleja relación entre los "formantes legales" del sistema, mientras que los formantes legales son todos aquellos elementos formativos que componen la aplicación cualquier regla.

El segundo problema con la metodología de Schlesinger es que se concibe sobre el uso de lo que puede llamarse una tecnología patológica: Se pide a los encuestados para resolver el problema jurídico, el conflicto. Esto significa que esta metodología no es adecuada para tratar de encontrar convergencia o divergencia entre los institutos jurídicos comparables o conceptos abstractos.

\footnotetext{
${ }^{13}$ Schlessinger, Rudolf, B. et al. Comparative Law Cases, Text, Materials. New York: New York Foundation Press, 1998.

${ }^{14}$ Sacco, Rodolfo. Legal Formants..., 1991.
} 


\subsection{La Metodología funcional}

El método funcional fue propuesto por Zweigert y Kötz ${ }^{15}$ en 1971. En realidad, la propuesta no pasaba de una afirmación genérica de principio y no contenía cualquier elemento de una teoría metodológica completa, pero en el momento de fuertes discusiones metodológicas fue suficientemente apelativa para un número significativo de estudiosos del Derecho. Como señala Micaels ${ }^{16}$, esa adhesión masiva ha hecho aparecer una multitud de técnicas de comparación funcional, que se desarrollarán de forma inorgánica y no teorizada.

Según Michaels, posición a la que nos adherimos, la clave del método funcional de Derecho comparado debe ser sacada dentro los conceptos sociológicos de funcionalidad y, de entre ellos, lo que ha tenido más y con mejores resultados en investigación de derecho comparado ha sido la de equivalencia funcional.

La equivalencia funcional puede sistematizar-se en siete funciones de equivalencia ${ }^{17}$ que son esenciales para el Derecho Comparado en el entorno metodológico: 1) la función epistemológica de la comprensión de las normas legales y las instituciones, 2) la función comparativa de lograr la comparabilidad, 3) la función de presunción de sobresalir en la similitud, 4) la función de formalización de la construcción del sistema 5) la función de evaluación de la determinación de la mejor ley, 6) la función universalizante de la preparación legal de unificación, y 7) la función crítica de proporcionar herramientas para la crítica del Derecho ${ }^{18}$.

Para Michaels la función primera de la función es epistemológica, o sea, la función de comprender el derecho. El método funcional permite comprender las instituciones legales no como construcciones doctrinales, sino como respuestas sociales a las instancias de los problemas, no como aislados, sino en su relación con el sistema entero, y más allá, a toda la sociedad. Para función comprender mejor esta función el camino es demostrar la diferencia entre el método factual o de Schlesinger o de caso, ya mencionado. El método factual utiliza casos que serán solucionados en distintos ordenamientos jurídicos y después hay que comparar las soluciones. Por veces este método es clasificado como funcional, pero realmente eso no es así. Con el

\footnotetext{
${ }_{16}^{15}$ Zweigert, Konrad. Kotz, Hein. An Introduction to Comparative Law. Oxford: Oxford, 1998.

${ }_{16}$ Michaels, Ralf. "The Functional Method of Comparative Law". En Reimann, Mathias., Zimmermann, Reinhard. (ed.).The Oxford Handbook of Comparative Law. Oxford: Oxford, 2006.

${ }^{17}$ Ibidem.

${ }^{18}$ Ibidem.
} 
método factual es posible encontrar similitudes o diferencias, pero no es posible saber si eso es una casualidad o una intencionalidad, mientras con el método funcional es posible construir cuestiones no litigiosas que muestran como la función de la institución jurídica se cumple integralmente y que otra institución, si alguna, cumple la misma función integralmente o parcialmente ${ }^{19}$.

La segunda función de la función es la de alcanzar la comparabilidad, es decir, la posibilidad de validar empíricamente una explicación funcional, a través de un referencial el tertium comparationis, el mismo una función. De hecho, las instituciones no son universales y por eso no pueden ser la constante necesaria para comparar, al contrario de las soluciones para una necesidad social, la función que, ese si, en principio, podrá ser universal o, al menos, generalizada en el universo en análisis. Esta es una cuestión esencial en que el dominio del concepto de "equivalente funcional" es de importancia fundamental. La tercera función es la de Praesumptio Similitudinis, o sea, hay que utilizar el equivalente funcional que permita cumplir la función de manera similar.

La cuarta función es sistemática, decir que es lo que permitirá construir un sistema de funciones y de necesidades. Es en este particular que el Derecho Comparado gana su naturaleza de disciplina científica típicamente empírica, ya que de la observación de ocurrencias especificas permite la generalización.

La quinta función es la función evaluativa. A partir de la sistematización echa por las cuatro funciones anteriores, es posible estudiar cual es la mejor (más eficiente) ley. Para ello, es necesario introducir una escala de valores que, todavía, no es ofrecida por el método funcional. La mejor practica en este particular será coger un criterio o escala de valuación que, en función del problema o necesidad, es más eficiente en su satisfacción.

La sexta función de la función es la función unificadora o armonizadora, esto es, ser un instrumento de unificación o armonización del Derecho, sea al nivel regional o universal. De hecho, el método funcional tiene la facultad de evidenciar las similitudes de soluciones entre distintos sistemas, aunque el camino sea diferente. Al final, si es verdad que este puede ser un argumento para no unificar, la verdad es que muestra, al mismo tiempo, que no es tan difícil armonizar - lo que al final del día interesa nos es tanto como llegar pero que si se llegue - esta es, la teleología implícita

\footnotetext{
${ }^{19}$ Ibidem.
} 
en lo concepto de Directiva de la Unión Europea o de variadas convenciones internacionales ${ }^{20}$.

$Y$, finalmente, la séptima función de la función es la función critica, al través de la cual se analiza críticamente un ordenamiento o solución jurídica ${ }^{21}$. Esta es una función de relevancia creciente, pues que es por esta función que se torna posible ultrapasar el argumento de la diversidad cultural muchas veces utilizado para justificar divergencias en las soluciones presentadas por distintos sistemas jurídicos para los mismos problemas - además, frecuentemente contradictorias y, por veces, inaceptables (lo decimos sin cualquier arrogancia intelectual occidental - imagínese la defensa de la esclavitud en el siglo XXI con base en la "cultura" dominante en la sociedad local, como autores que no honramos con referencia han hecho). En casos mucho menos dramáticos o graves, el método funcional permite, con la base de equivalente funcional, que tiene que considerar las diferencias culturales, jurídicas $\mathrm{u}$ otras, identificar las eficiencias e ineficiencias de las varias leyes o soluciones en comparación, actuando como un instrumento de mejoría de la menos eficaz.

\section{Derecho Comparado y Derechos Humanos, una sugerencia metodológica}

El dominio de los Derechos Humanos es un área científica en la que la investigación está creciendo exponencialmente. Eso son buenas noticias, aunque las razones determinantes puedan ser las malas noticias - menor respecto por los Derechos Humanos, migraciones forzadas y refugiados de guerra u otros.

El reto, pero también la oportunidad, que los estudiosos enfrentan en este particular es que, al final, el estudio de los Derechos Humanos es, o tiende a ser, un estudio pluridisciplinar: si el tema empieza por ser jurídico, pues que de un instituto jurídico hablamos, talvez en ninguna otra área jurídica encontramos la diversidad de interacciones presente en esta.

La investigación comparativa en Derechos Humanos puede utilizar cualquiera de los métodos que enumeramos, con las ventajas y desventajas de cada uno $\mathrm{y}$, en este particular, este dominio científico no es distinto de otras investigaciones jurídico-comparativas.

\footnotetext{
${ }^{20}$ Ver las conclusiones, sobre Derecho Europeo de la Propiedad en Caramelo Gomes, Jose L. The Law of Real Property in The European Community. A Comparative Study. Vol. PhD. Salford: University of Salford, 2002.

${ }^{21}$ Michaels, Ralf. The Functional Method ..., 2006.
} 
La existencia de normativo internacional, así como el universalismo de los Derechos Humanos, tiene una gran utilidad como tertium comparationis para el método conceptual. De la misma forma, la jurisprudencia internacional en esta materia puede funcionar como tertium comparationis para la metodología de caso o hasta en la metodología funcional. Así, en el punto de partida, todas las metodologías presentadas en este artículo pueden aplicarse al estudio comparativo de los Derechos Humanos.

En la medida en que la temática es especialmente influenciada por el contexto, las metodologías comparativas que investigan la ley en funcionamiento son particularmente adecuadas: el método de caso y el método funcional. El primero de ellos será particularmente adecuado cuando sea necesario averiguar el aspecto crucial la tutela jurisdiccional efectiva y el segundo cuando sea importante averiguar la existencia de equivalentes funcionales que, por veces, quedan poco visibles - será que la Ley de la Pureza de la Cerveza bávara del sículo XIV/XV, que hablaba del divino derecho de beber cerveza, en consecuencia de la Peste Negra, no consagraba ya el Derecho à vida o a la salud?

\section{Referencias Bibliográficas}

Caramelo Gomes, José Luis. The Law of Real Property in The European Community. A Comparative Study. Vol. PhD. Salford: University of Salford, 2002.

David, René.; Brierley, John E. Major legal systems in the world today: an introduction to the comparative study of law: Simon and Schuster. s.d., 1978.

ERP, J. European Private Law Postmodern Dilemmas and Choices. Towards a Method of Adequate Comparative Legal Analysis. s.d., EJCL. 1999.

Glenn, H.Patrick. "Aims of comparative law". In: Smits, J.M., (ed.). Elgar Encyclopedia of Comparative Law, Cheltenham: Elgar Publishing; 2014.

Jayme, Erik. "Identité culturelle et intégration: Le Droit international privé postmoderne". Recueil des Cours, Hague Academy of International Law, 1995.

Legrand, Pierre. Sens et non-sens d'un Code civil européen. RIDC, 1996, pp.779-812.

Michaels, Ralf. "The Functional Method of Comparative Law". En: Reimann, M.; Zimmermann, R. (ed.).The Oxford Handbook of Comparative Law. Oxford: Oxfrd, 2006. 
Pieters, D. "Functions of comparative law and practical methodology of comparing". Syllabus Research Master in Law. 2009.

Sacco, Rodolfo. "Legal Formants, A Dynamic Approach to Comparative Law". American Journal of Comparative Law, 1991.

Schlessinger, Rudolf B. et al. Comparative Law Cases, Text, Materials. New York: New York Foundation Press, 1998.

Van der Mensbrugghe, François. L'utilisation de la méthode comparative en droit européen. Namur: Presses universitaires de Namur, 2003.

Zweigert, Konrad. y Kotz, Hein. An Introduction to Comparative Law. Oxford: Oxford, 1998. 